\title{
CARACTERIZACIÓN DE NIÑOS OPERADOS POR LABIO Y PALADAR HENDIDO EN LA ORINOQUÍA COLOMBIANA ENTRE 2008 Y 2014
}

Libardo Vacca-Devia', Julián Quintero-Vacca², Norton Pérez-Gutiérrez³ Sonia del Pilar Rubio-Caicedo ${ }^{4}$, Emma Isabel Rodríguez Darabos ${ }^{5}$

\section{Resumen}

La patología congénita de niños con labio y paladar hendido es frecuente en la región, pero no ha sido caracterizada en la Orinoquía. El objetivo de este estudio fue describir las características de los niños intervenidos en un programa quirúrgico de labio y paladar hendido en Villavicencio. Tipo de estudio: Corte transversal descriptivo. Criterios de inclusión: menores de 18 años operados por labio y paladar hendido. Escenario: Hospital Departamental de Villavicencio, Colombia. Temporalidad: enero de 2008 a junio de 2014. Se realizó una evaluación descriptiva de la distribución de las variables, análisis bivariado y un mapa de georreferenciación del sitio de procedencia de los pacientes. Resultados: Se encontraron 340 registros de niños intervenidos quirúrgicamente por labio y paladar hendido, mayormente de sexo masculino. La mayoría de los casos fueron procedentes de Villavicencio, pero también de otros municipios y departamentos circundantes. El $49 \%$ de los procedimientos fueron primarios y las niñas fueron intervenidas más tempranamente. Conclusión: El estudio permite caracterizar el número considerable de pacientes intervenidos, la mayoría de ellos de forma temprana, especialmente en los casos cercanos de Villavicencio y el Meta, aunque puede mejorarse la oportunidad a los primeros meses de vida.

Palabras clave: labio leporino, fisura del paladar, niño, misiones médicas, Colombia.

${ }^{1}$ Cirujano maxilo-facial del Hospital Departamental de Villavicencio. Profesor de Medicina de la Universidad Cooperativa de Colombia.

${ }^{2}$ Estudiante de Medicina de la Universidad Cooperativa de Colombia.

${ }^{3}$ Médico y cirujano; cirujano general; subespecialista en Medicina Crítica y Cuidado Intensivo. Coordinador de las UCI del Hospital Departamental de Villavicencio. Director del Grupo de Investigación de Villavicencio (GRIVI).

${ }^{4}$ Gerente de la Fundación Sueños y Sonrisas.

${ }^{5}$ Excoordinadora de calidad del Hospital Departamental de Villavicencio. Gerente de A\&R gestión. Subdirectora del Grupo de Investigación de Villavicencio (GRIVI). 


\section{CHARACTERIZATION OF CHILDREN WHO HAD SURGERY OF CLEFT LIP AND PALATE IN THE COLOMBIAN ORINOQUIA BETWEEN 2008 AND 2014}

Libardo Vacca-Devia, Julián Quintero-Vacca, Norton Pérez-Gutiérrez, Sonia del Pilar Rubio-Caicedo, Emma Isabel Rodríguez Darabos

\section{Abstract}

Cleft lip and palate is the most frequent congenital pathology but it has not been characterized in the Colombian Orinoquia. The aim was to describe the characteristics of children that had surgery in a program for cleft lip and palate in Villavicencio. Design: cross-sectional descriptive trial. Children under 18 years old that had cleft lip and palate surgery at Hospital Departamental de Villavicencio from January 2008 to June 2014 were included. Distribution evaluation of the variables was performed, as well as a map with the housing location of patients Results: Three hundreds and forty records of cleft lip and palate surgeries were reviewed, mainly male children. Most of the cases were from Villavicencio, but also from other towns and states around. Fortynine percent were primary surgeries and female children had interventions earlier. Conclusions: the study established the considerable number of patients surgically operated, most of them in an early way, especially when they were close to Villavicencio and Meta, but opportunity can be improved to early months of life. 


\section{CARACTERIZAÇÃO DE CRIANÇAS OPERADOS POR FENDA LABIAL E PALATINA NA ORINOQUIA COLOMBIANA ENTRE 2008 E 2014}

Libardo Vacca-Devia, Julián Quintero-Vacca, Norton Pérez-Gutiérrez Sonia del Pilar Rubio-Caicedo, Emma Isabel Rodríguez Darabos

\section{Resumo}

A patologia congénita em crianças com fenda labial e palatina é frequente na região, mas no tem sido caracterizada na Orinoquia colombiana. Objetivo: descrever as características das crianças intervindos num programa cirúrgico de fenda labial e palatina na cidade de Villavicencio. Tipo de estudo: descritivo-transversal. Critérios de inclusão: pessoas menores de 18 anos operados por fenda labial e palatina. Lugar: Hospital Departamental de Villavicencio, Colômbia. Tempo: janeiro do 2018 até junho do 2014. Realizou-se uma avaliação descritiva da distribuição das variáveis, análise bivariada e mapa de georreferenciamento no ponto de procedência dos pacientes. Resultados: encontrou-se 340 registros de crianças intervindos cirurgicamente pela fenda labial e palatina, sendo maior os processos no sexo masculino. A maioria dos casos foram feitos em Villavicencio, além de outras cidades menores. O 49\% dos procedimentos foram primários e as meninas foram intervindas cedo. Conclusões: o estudo permite caracterizar o número de casos, pacientes intervindos, na maioria deles tiveram a cirurgia cedo, especialmente nos casos perto da cidade de Villavicencio e no estado do Meta, embora podem melhorar-se a oportunidade nos primeiros meses de vida. 


\section{Introducción}

Las anomalías congénitas son una causa importante de mortalidad infantil. Las malformaciones cráneo-faciales constituyen entre el 10-15\%, y entre ellas, la hendidura oral se presenta entre 1:500-700 nacimientos. Hay gran variabilidad geográfica en la presentación del labio y paladar hendido, al igual que en los estratos socioeconómicos; igualmente, hay variantes dela presentación y es una condición que genera vergüenza al paciente y la familia, y mayores riesgos para el bebé, especialmente en algunas comunidades como las indígenas. Existe documentación de su aparición ancestral en amerindios mediante la orfebrería indígena $(1,2)$. Adicionalmente, es frecuente la asociación con otras anomalías mayores que tienen gran impacto en la mortalidad (3).

Las deformidades con hendidura del labio pueden ser uni o bilaterales, asociarse con o sin paladar hendido y son la malformación cráneo-facial más frecuente (4). Se originan por defecto en la migración y fusión de los procesos maxilares y el proceso nasal medial. Tienen múltiples factores que han sido estudiados, entre ellos cromosómicos, familiares, alimentarios y ambientales o pueden estar asociados con algún síndrome congénito.

Son frecuentes en países de Asia como China, según los registros de nacimiento, en donde se ha encontrado con una proporción entre $0,1 \%$. Son más frecuentes en embarazos múltiples y hay variaciones de la presentación entre hombres (labio y paladar) y mujeres (paladar); la edad gestacional (37 semanas), la multiparidad

y el bajo peso $(<1,5 \mathrm{~kg})$ son factores de riesgo para la enfermedad (5).
La intervención temprana es definitiva para los resultados estéticos y funcionales. Se han descrito algunas experiencias en el manejo quirúrgico en regiones de Colombia como Manizales, Medellín y Cali, pero no en Villavicencio $(4,6)$.

En el Hospital Departamental de Villavicencio, el mayor centro de referencia de la Orinoquía colombiana, se ha desarrollado un programa de labio y paladar hendido desde hace más de 18 años. Inicialmente, con el apoyo del programa de Healing the Children (HTC) - Michigan, actualmente es liderado por cirujanos de la región (7).

Sin embargo, no se encuentran muchos artículos en la literatura científica sobre esta vasta experiencia, ni sobre sus hallazgos y los resultados. En una búsqueda en bases de datos internacionales, de revistas médicas y de la región, solo se encontraron dos artículos que mencionaran el problema en Villavicencio $(8,9)$ No se encontró ningún resultado al aplicar los descriptores de ciencias de la salud "Cleft" AND "Colombia” en Pubmed. La misma búsqueda aplicada en los títulos de los artículos arrojó 35 resultados, de los cuales 21 fueron pertinentes, pero solo uno de los trabajos incluyó pacientes de Villavicencio de un estudio descriptivo multicéntrico (7).

La cuantificación de los casos de labio y paladar hendido es relevante a fin de evaluar la magnitud del problema en la región. Esto permite caracterizar los casos con el propósito de diseñar estrategias que permitan la atención temprana de la patología. Es así como se plantean las siguientes preguntas: ¿cuáles son las características de los niños intervenidos 
por labio y paladar hendido en el Hospital Departamental de Villavicencio? ¿A qué edad suelen realizarse los primeros procedimientos en niños con labio y paladar hendido en Villavicencio?

El objetivo del estudio fue describir las características de los niños intervenidos en un programa quirúrgico de labio y paladar hendido en Villavicencio.

\section{Metodología}

Se diseñó un estudio de corte transversal descriptivo, de carácter retrospectivo, a partir de una base de datos quirúrgica de pacientes operados por labio y paladar hendido en el Hospital Departamental de Villavicencio, Colombia, entre enero de 2008 y junio de 2014. El criterio de inclusión fueron los registros de pacientes menores de 18 años de edad, intervenidos quirúrgicamente por labio o paladar hendido en el periodo de estudio. Se excluyeron del análisis los registros que no tuvieran los datos de las variables seleccionadas para el estudio. La muestra fue seleccionada por método no probabilístico, por conveniencia, no apareada de casos continuos de acuerdo con los criterios de inclusión y exclusión.

Las variables demográficas y clínicas de los pacientes fueron exportadas a Excel para su depuración y analizadas con el programa SPSS versión 15, a fin de realizar las pruebas de estadística descriptiva y bivariada. No se recolectaron datos de la familia ni de otras variables asociadas con la etiología de la enfermedad. El mapa de georreferenciación fue diseñado en el programa Epi Info versión 7 con los datos de la vivienda o procedencia de la familia del paciente.

Se analizaron las medidas de frecuencia y proporción para las variables categóricas y de tendencia central y distribución en las variables continuas. Se realizó comparación de las variables dependientes (tipo de reparo) e independientes en búsqueda de patrones de asociación con los tipos de malformación y el sexo, así como la edad a la que fueron intervenidos los niños de Villavicencio en contraste con los demás, y se definió como significancia cuando la $\mathrm{p}$ fue $<0,05$.

\section{Aspectos éticos}

Se trata de un estudio retrospectivo que involucra la revisión de bases de datos, que no implica ningún riesgo para los sujetos. Igualmente, se mantuvo la confidencialidad de la identidad de los pacientes y sus familias, según lo establecido en la Resolución 8430 de 1993 del Ministerio de Salud. El trabajo fue presentado y aprobado por el comité de investigación de la Facultad de Medicina de la Universidad Cooperativa de Colombia y del Hospital Departamental de Villavicencio y se otorgó una exención del consentimiento informado por el diseño y sin riesgo para los pacientes.

\section{Resultados}

Se revisaron los registros de 439 pacientes, entre los cuales 340 cumplieron con los criterios de inclusión y exclusión (tabla 1). Se encontró una mayor proporción de niños (55\%) y en general las edades fueron en promedio de 4,59 años (DE 4,98) (figura 1). 
INVESTIGACIONES ANDINA No. 34 Vol. 19

Tabla 1. Características demográficas de los niños operados por labio o paladar hendido en el Hospital Departamental de Villavicencio entre 2009-2014

\begin{tabular}{|c|c|c|}
\hline & $\mathbf{n}$ & $\%$ \\
\hline $\mathrm{N}$ & 340 & 100 \\
\hline \multicolumn{3}{|l|}{ Sexo } \\
\hline Niños & 186 & 54,71 \\
\hline Niñas & 154 & 45,29 \\
\hline \multicolumn{3}{|l|}{ Departamento } \\
\hline Boyacá & 2 & 0,59 \\
\hline Casanare & 7 & 2,06 \\
\hline Guainía & 3 & 0,88 \\
\hline Guaviare & 81 & 23,82 \\
\hline Meta & 235 & 69,12 \\
\hline Vaupés & 1 & 0,29 \\
\hline Vichada & 11 & 3,24 \\
\hline \multicolumn{3}{|l|}{ Técnica } \\
\hline Primario labio y nariz & 70 & 19,94 \\
\hline Primario labio bilateral y nariz & 15 & 4,27 \\
\hline Paladar primario & 87 & 24,79 \\
\hline Reparo de fístula & 64 & 18,23 \\
\hline Revisión labio y nariz & 77 & 21,94 \\
\hline Hendidura secundaria & 13 & 3,70 \\
\hline Injerto hueso alveolar & 23 & 6,55 \\
\hline Otro & 2 & 0,57 \\
\hline
\end{tabular}

Fuente: Elaboración propia con base en los resultados.

La mayoría de los pacientes fueron del departamento del Meta (69\%), seguido por el Guaviare (24\%), pero se intervinieron niños procedentes de toda la región de la Orinoquía y sus alrededores (figura 2).
Los niños del Meta fueron mayormente del municipio de Villavicencio, y de estos la mayor parte estuvieron concentrados en la Comuna 3 (43\%) (figura 3). 


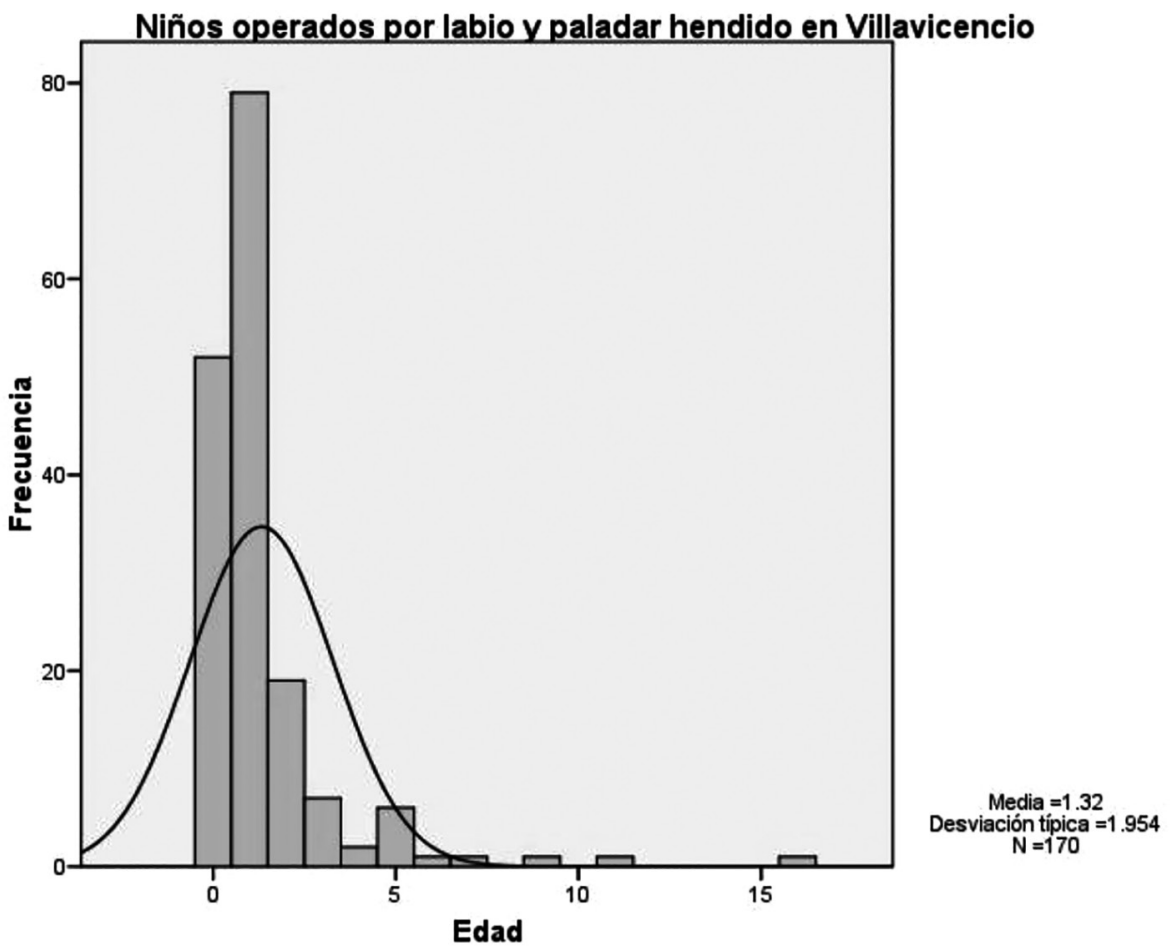

Figura 1. Frecuencia y rango de edad de los niños operados por labio y paladar hendido en Villavicencio (2008-2014)

Fuente: Elaboración propia con base en los resultados.

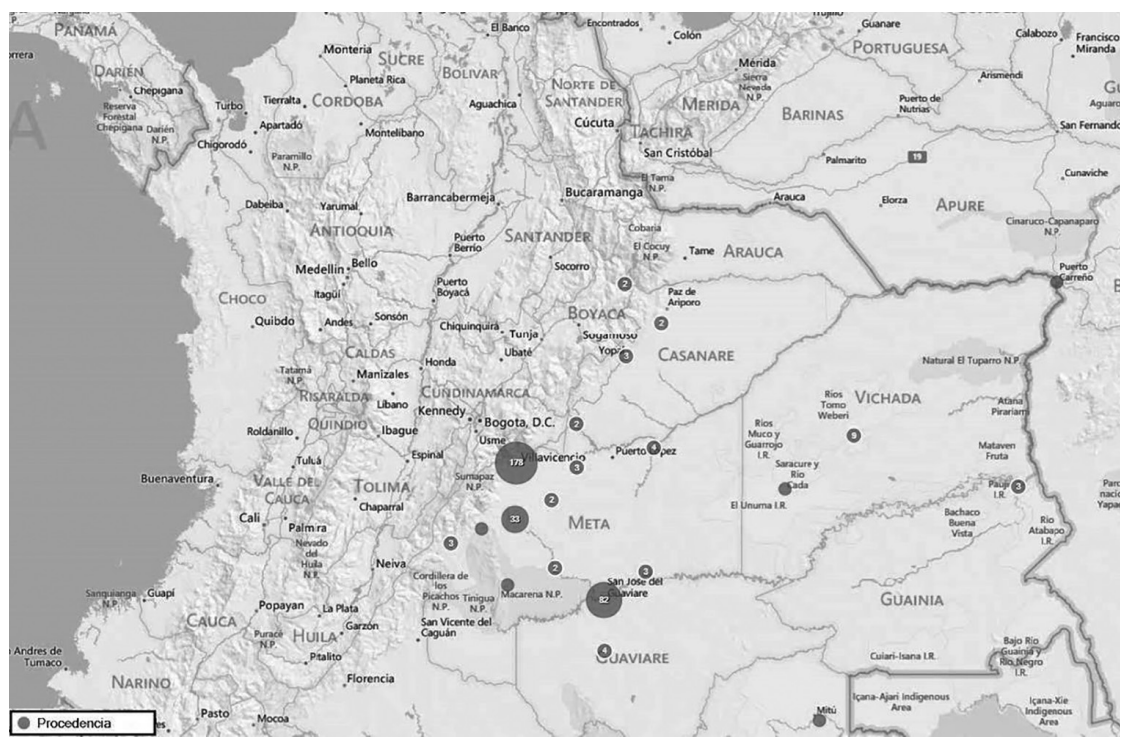

Figura 2. Mapa epidemiológico con la procedencia de los niños operados por labio y paladar hendido en Villavicencio (2008-2014).

Fuente: Elaboración propia. Diseñado con el programa Epi Info 7. 


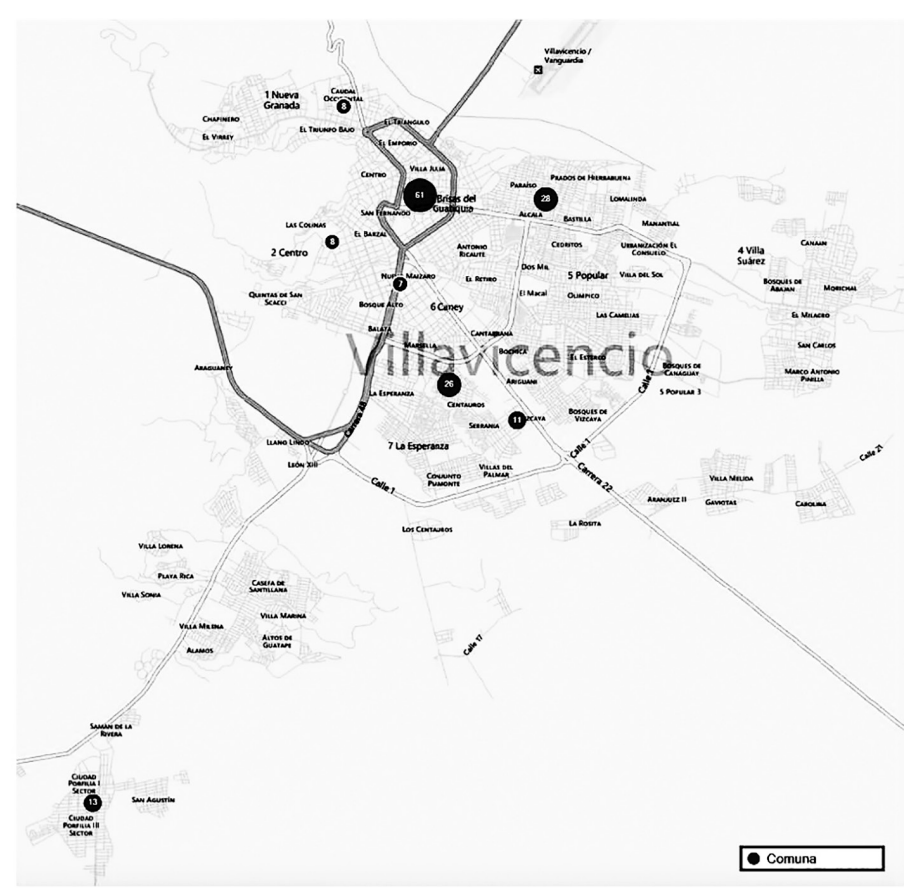

Figura 3. Distribución geográfica de la comuna de procedencia de los niños operados por labio y paladar hendido de Villavicencio (2008-2014).

Fuente: Elaboración propia. Diseñada con el programa Epi Info 7.

Las intervenciones quirúrgicas se realizaron desde el primer año y el $40 \%$ fueron en menores de 2 años. Se intervinieron 48,6 casos por año en promedio (rango 5-70) y el mayor número de intervenciones fue en el año 2011 y el menor en el 2008 (figura 4). Se realizaron 351 procedimientos y el $50 \%$ de estos fueron primarios (tabla 1). La mayoría de los procedimientos fueron corrección de fisura del paladar primario (25\%), seguido por revisión de labio y nariz, reparo primario de labio y nariz y reparo de fístula $(22,20$ y $18 \%$, respectivamente).

En el análisis bivariado se encontró que las niñas fueron intervenidas más tempranamente que los niños en todos los procedimientos en general $(4,06 \pm$ 4,87 vs. 5,02 $\pm 5,04$ años), así como en los procedimientos primarios $(1,28 \pm$ 1,45 vs. 1,37 $\pm 2,4$ años), (figuras 5, 6 y 7). Asimismo, fueron intervenidos más tempranamente los niños del departamento del Meta y más tardíamente los del Guaviare y Guainía, tanto en los procedimientos primarios como en las revisiones (figuras 8 y 9). En los dos departamentos con mayor número de pacientes, Meta y Guaviare, la proporción de hombres fue un poco mayor $(54,5 \mathrm{y}$ $61,7 \%)$, al igual que en el municipio de Villavicencio (52,2\%). 


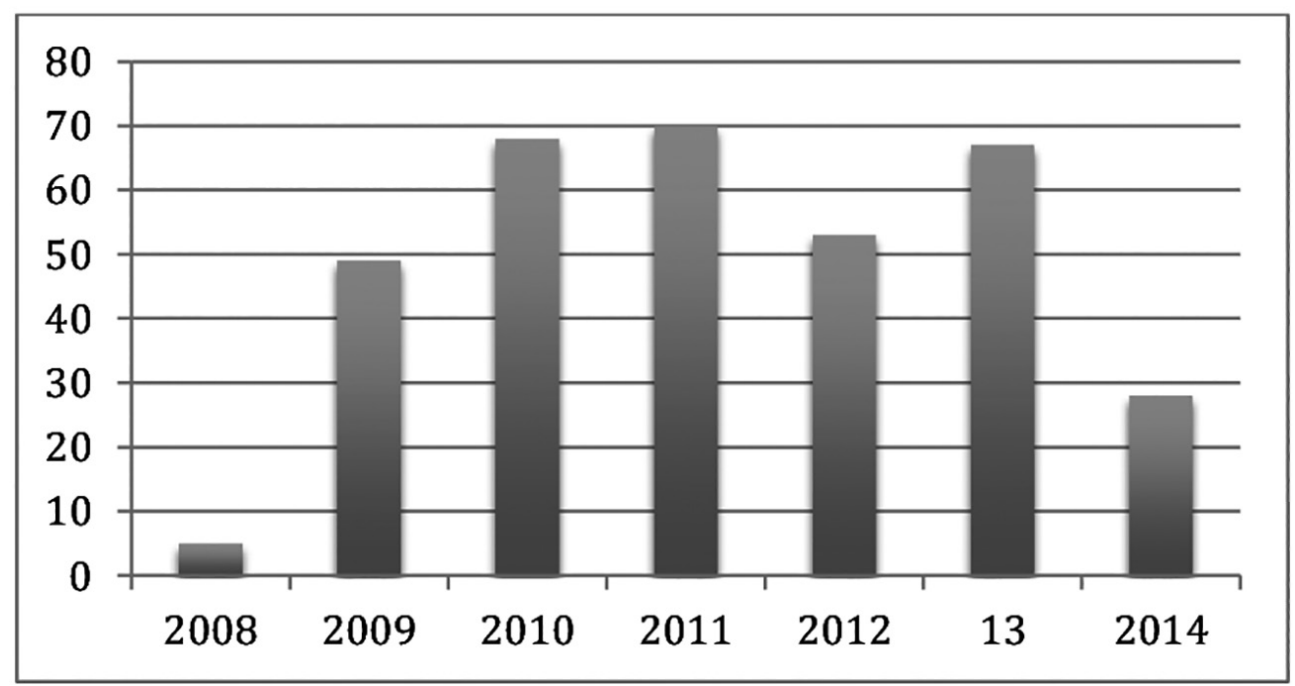

Figura 4. Casos intervenidos por año en niños con labio y paladar hendido en Villavicencio (2008-2014). Fuente: Elaboración propia con base en los resultados.

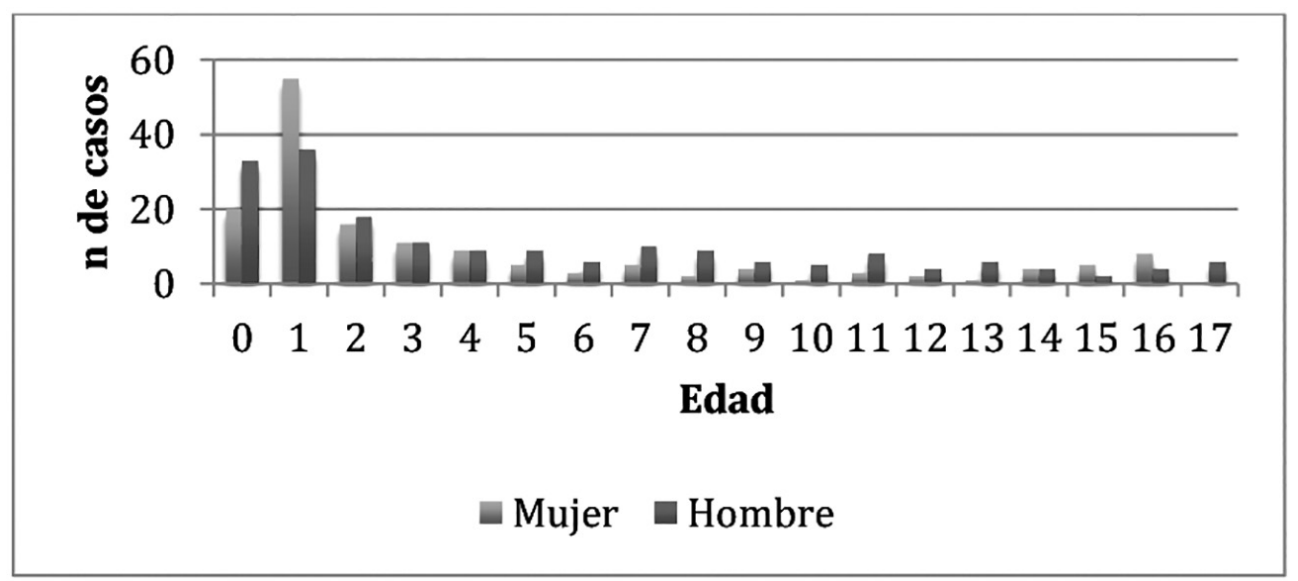

Figura 5. Distribución de los casos operados de labio y paladar hendido por edad y sexo en Villavicencio (2008-2014).

Fuente: Elaboración propia con base en los resultados. 


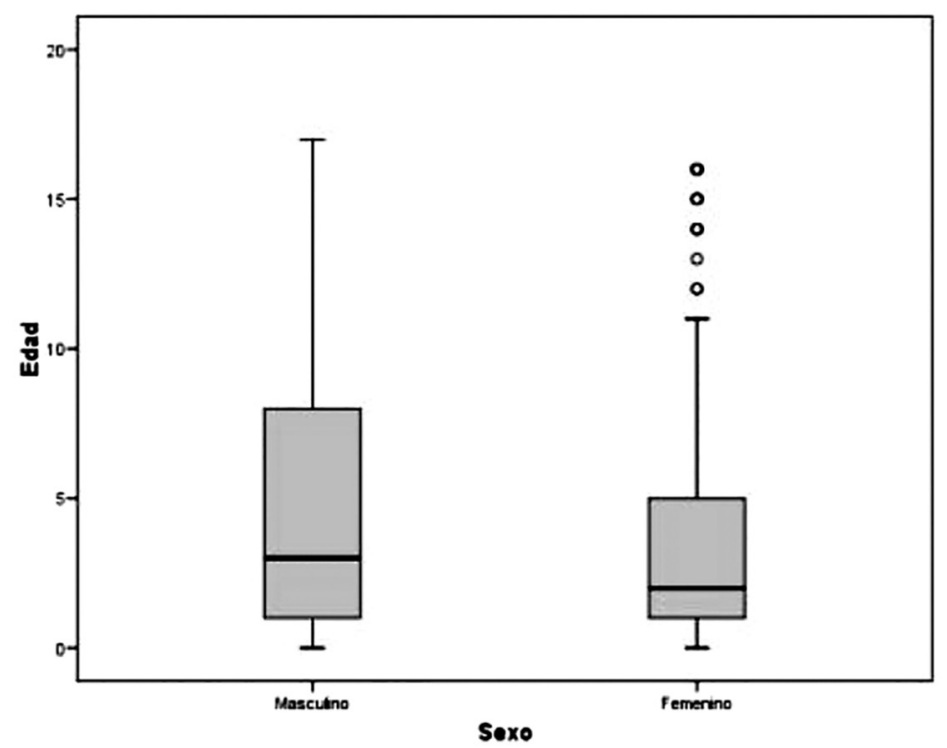

Figura 6. Comparación de la edad por sexo en niños intervenidos quirúrgicamente por labio y paladar hendido en Villavicencio (2008-2014). Las niñas son intervenidas más tempranamente que los niños $(p<0,05)$.

Fuente: Elaboración propia con base en los resultados.

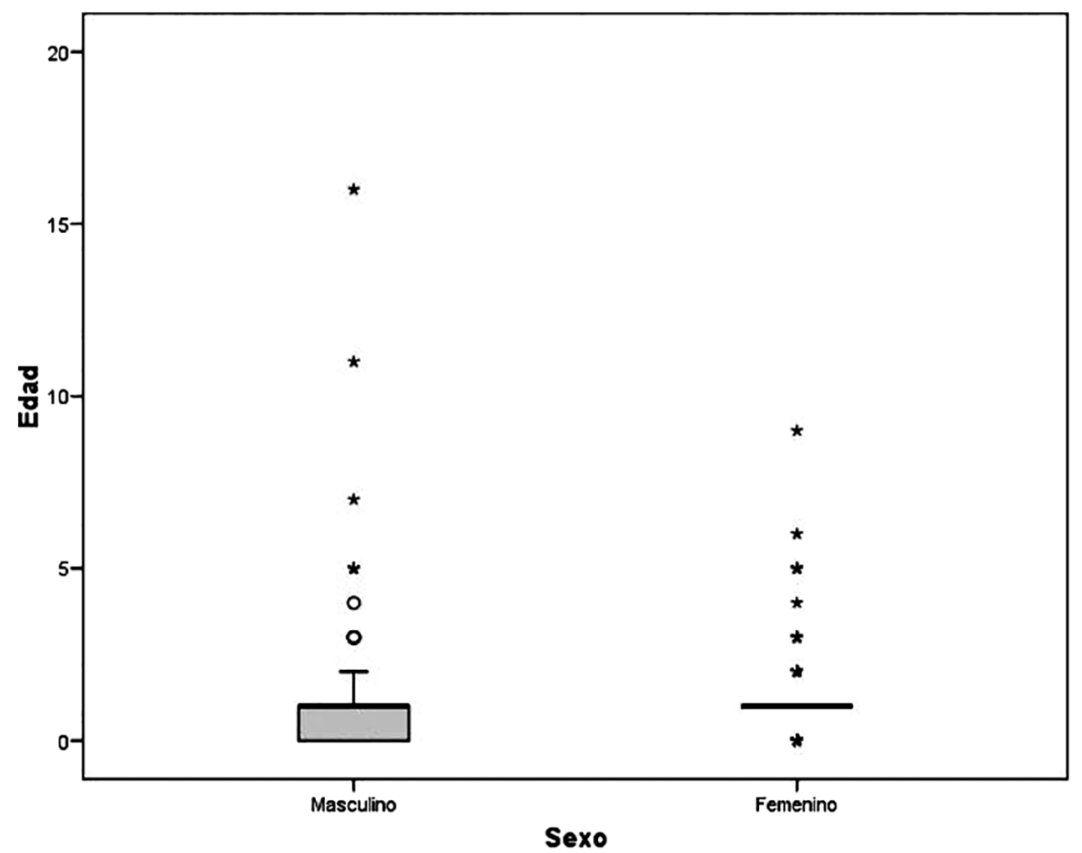

Figura 7. Comparación de la edad para el reparo primario por sexo en niños operados por labio y paladar hendido en Villavicencio (2008-2014). Aunque se encontraron algunos casos tardíos y no hubo diferencias significativas, las niñas tienden a ser intervenidas más tempranamente $(p=0,2)$.

Fuente: Elaboración propia con base en los resultados. 


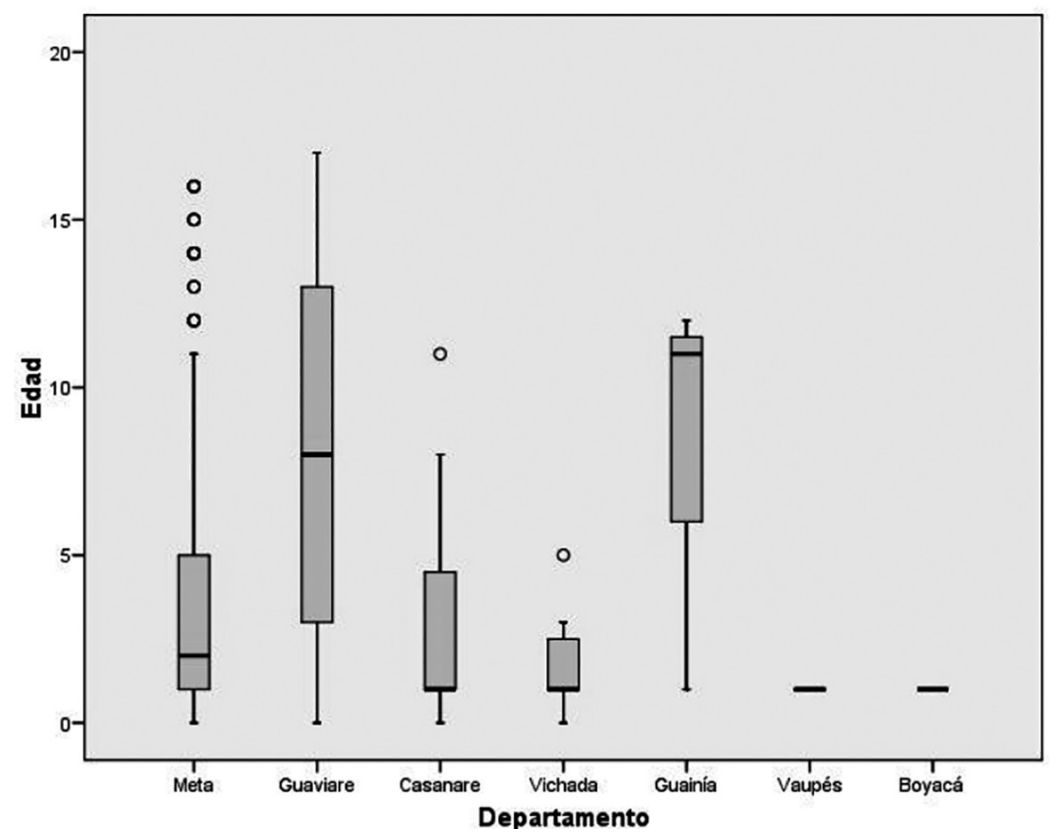

Figura 8. Edad por departamentos en cirugía de labio y paladar hendido en Villavicencio (2008-2014). Los niños del Meta fueron operados más tempranamente $(p<0,05)$.

Fuente: Elaboración propia con base en los resultados.

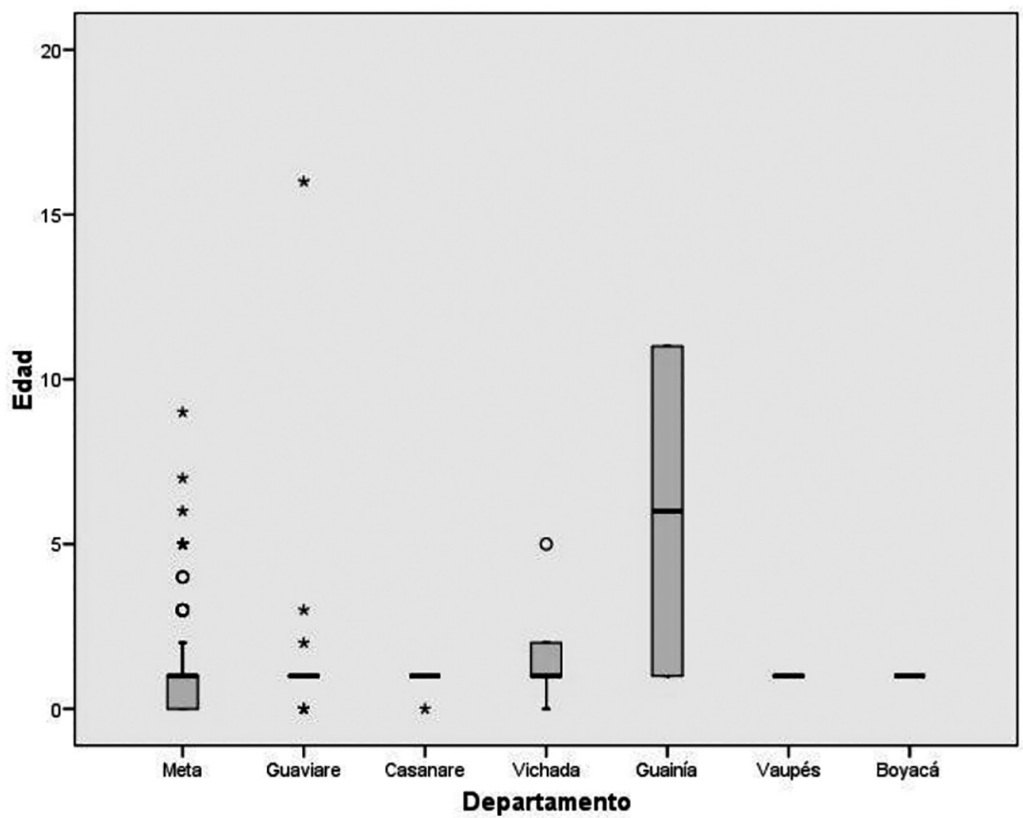

Figura 9. Edad por departamento en los procedimientos primarios de labio y paladar hendido en Villavicencio (2008-2014).

Fuente: Elaboración propia con base en los resultados. 
Los reparos primarios del paladar (desenlace) se hicieron más frecuentemente en niñas $(57,8 \% v s .43,5 \%)$, mientras que la revisión de labio y nariz lo fue en niños (71\% vs. 29\%). Ambas diferencias fueron significativas $(p<0,05)$. El 23,6\% de los procedimientos en hombres fueron primarios, mientras que en las mujeres fue el $25,4 \%(p<0,02)$.

La proporción de reparos primarios fue mayor en Villavicencio 58,2\% vs. 41,8\%). El 57,9\% de los casos primarios fueron del Meta, y entre ellos, el 72,8\% fueron de Villavicencio. El 52,4\% de los casos del municipio de Granada, Meta, fueron primarios. La edad promedio de los reparos primarios fue menor en niños de Villavicencio que en el resto de municipios ( $1,1 \pm 1,3$ vs. $1,6 \pm 2,6$ años $)$.

\section{Discusión}

Las deformidades cráneo-faciales son los defectos congénitos más comunes y requieren un manejo temprano y multidisciplinario. Algunos de estos pueden estar asociados a otras anomalías (22\%) y requieren intervenciones mayores, hospitalizaciones múltiples y estancia prolongada (10).

La multicausalidad del evento dificulta las estrategias de prevención de esta condición que tiene un gran impacto social y clínico (11). Los factores genéticos y familiares han sido estudiados $(12,13)$. No se ha podido demostrar el papel etiológico de otros factores dietarios como la ingesta de nitrosaminas (14). Un estudio que analizó una gran base de datos de nacimientos en hospitales de América Latina (solo 3 eran colombianos) no encontró una asociación con la multiparidad $(+4)$, a pesar de la creencia popular (15). El efecto protector dela suplementación de ácido fólico para la prevención de las fisuras orales ha sido informado en algunos estudios, incluso a dosis altas $(16,17)$. La epilepsia y los anticonvulsivantes se han identificado como factores para las malformaciones congénitas, entre ellas, las orofaciales (18). La altura sobre el nivel del mar es otro factor mencionado en la literatura (19).

Un estudio europeo que hace un análisis de las estadísticas de una gran base de datos estima la prevalencia de la hendidura del labio en 9,92 por 10.000 nacimientos con o sin compromiso del paladar (20). Países como el Reino Unido han centralizado la atención de esta patología, lo cual ha permitido una intervención temprana en los 2 primeros años de vida (10). En este mismo estudio, se encontró que los defectos del labio son corregidos tempranamente (primeros 5 meses), mientras que los del paladar en los primeros 2 años. En Finlandia, donde se lleva un registro único nacional de nacimientos, reportaron, ya hace varios años, una alta incidencia de labio y paladar hendido (21). Países como México y Brasil han divulgado también su experiencia en la literatura (22).

Las publicaciones nacionales describen una prevalencia mayor, 1 por 500-700 nacimientos, mientras que la literatura internacional la describe en 1 por 1.000 , $(3,18,23,24)$. Otras ciudades en Colombia han publicado su experiencia con programas similares (24-27). La principal institución de atención materno-pediátrica en el país ha publicado su amplia experiencia, incluso una guía de manejo. Los factores sociales como la pobreza y el 
tipo de aseguramiento tienen un impacto en el manejo en nuestro medio (25).

Un estudio multicéntrico sobre la experiencia de una misión médica ( $>18$ años), con 2.410 cirugías de labio y paladar hendido, incluye pacientes de Villavicencio (7). En esta descripción resaltan algunos hallazgos, como un 39\% de deformidades bilaterales, predominancia de las hendiduras izquierdas en las presentaciones incompletas y alta incidencia de hendiduras aisladas en el paladar blando. Si bien se podría pensar en un sesgo de selección, debido a la metodología de reclutamiento de los pacientes, este trabajo describe un tercio de antecedentes familiares, mientras que otro estudio en Medellín encontró 17,7\% del lado paterno y $21,6 \%$ del lado materno (26).

En el presente estudio se revisa la casuística quirúrgica registrada en una base de datos de niños con labio y paladar hendido en un hospital de Villavicencio que es centro de referencia para una amplia zona de Colombia. Allí confluyen pacientes de la Orinoquia y Amazonía colombiana que consultan de forma espontánea o remitidos de otras instituciones. Las características de esta zona, de población dispersa (4,6 habitantes $\left./ \mathrm{km}^{2}\right)$, gran extensión y pocas vías de comunicación, dificultan el acceso a los servicios de salud (28).

La mayor proporción de población de niños del Meta y de Villavicencio atendidos en el estudio se explica por un mejor acceso a los servicios de salud por la cercanía a la institución. La diferencia en la edad más temprana para las niñas en los casos primarios y para los casos del Meta y de Villavicencio son hallazgos incidentales que merecen análisis posteriores, pero que podrían ser explicados inicialmente por factores culturales (priorización a las niñas) o dificultades en el acceso a los servicios de salud. Otras publicaciones colombianas han evaluado la frecuencia de presentación de esta patología, para lo cual se debe tener en cuenta que se trató de centros de referencia de las instituciones del mayor nivel de complejidad, lo cual puede magnificar el cálculo de la incidencia en recién nacidos en esas instituciones pero no ser reflejo del problema poblacional $(3,4,23-27,29-34)$. En este caso, los registros nacionales de recién nacidos, como el realizado en Finlandia, puede ser de mayor precisión (21).

Se encontraron diferencias en la distribución del sexo, principalmente en los casos primarios, ya que las niñas fueron atendidas de forma más temprana. La mayor concentración de casos procedentes de Villavicencio y Meta se da por la posibilidad de acceso de estos a la institución, pero se debe tener en cuenta que esta también es el principal centro de atención de mayor complejidad de la población de toda la región para la solución de este problema. El alto número de casos primarios da cuenta de que sigue habiendo un flujo de casos nuevos al programa.

No se conoce la incidencia de esta enfermedad en la institución ni en registros de recién nacidos en la región, y el diseño de este estudio no permite calcularlo, lo cual plantea un nuevo tema para futuras investigaciones en esta línea.

El bajo nivel de conciencia de este problema, incluso en comunidades que tienen una alta prevalencia, dificulta su manejo quirúrgico, psicológico y la rehabilitación 
social y no tiene prioridad en los programas gubernamentales.

Esta etapa inicial deja algunas preguntas de investigación (entre otras), para futuros estudios:

- ¿Cuál es la incidencia de niños recién nacidos con labio y paladar hendido en Villavicencio y el Meta?

- ¿Hay diferencias en la incidencia con otros municipios y departamentos de la región?

- ¿Cuáles son los factores de riesgo presentes en estos niños (familiares, ambientales, genéticos, etc.)?

- ¿Cuáles son los costos (directos e indirectos) en la atención quirúrgica e integral de esta patología?

- ¿Cuáles son las medidas de prevención más efectivas y sus costos derivados?

- ¿Cuáles son los resultados quirúrgicos, escolares, funcionales, nutricionales y psicológicos en los niños intervenidos quirúrgicamente de forma más temprana?

Finalmente, los niños con fisuras de labio y paladar han sido atendidos por más de 20 años en la Orinoquía colombiana a través de programas de jornadas de voluntarios de HTC-Míchigan. Actualmente, el programa es liderado por cirujanos y voluntarios de la región y ha permitido la continuidad y el enfoque multidisciplinario de rehabilitación planteado en los principios por Macintosh y otros (7). Aún falta mucho camino por recorrer, pero esta transferencia de experiencias y conocimiento ha llevado alegrías y sonrisas a muchos niños y sus familias que, de no haber sido así, no hubieran sido posibles.

La discusión sobre el papel de las misiones quirúrgicas en regiones apartadas debería continuar, con mayor participación de quienes han sido receptores de las visitas, pero se escapa al objetivo de esta investigación y merece una revisión en otro aparte (35).

El estudio tiene limitaciones debido a su diseño descriptivo y retrospectivo, a la ausencia de otras variables de importancia en los registros clínicos, pero en su intención exploratoria se pueden encontrar datos útiles para determinar fenómenos como la procedencia y la caracterización de la población de estudio, que son de utilidad para orientar la continuidad del programa e incluso ayudar a orientar esfuerzos gubernamentales para manejar el problema desde las autoridades de salud pública.

Se requiere ajustar la estructura de la base de datos y la sistematización de la recolección de la información con otras variables de importancia en el tema, para análisis futuros y seguimiento.

\section{Conclusiones}

El estudio permite establecer el número considerable de pacientes atendidos en el programa quirúrgico de labio y paladar hendido, con una intervención temprana del $49 \%$ de los casos, especialmente en los que se han presentado cerca de Villavicencio y el Meta, aunque puede mejorarse la oportunidad a los primeros meses de vida. 


\section{Referencias}

1. Pachajoa $H$, Hernandez-Amaris $M F$, Porras-Hurtado GL, Rodriguez CA. Siamese twins with craniofacial duplication and bilateral cleft lip/palate in a ceramic representation of the Chimú culture (Peru): a comparative analysis with a current case. Twin Res Hum Genet. 2014 Jun;17(3):211-4.

2. Orticochea M. The harelipped king: a pre-Colombian ceramic statue over 2000 years old. Br J Plast Surg. 1983 Jul; 36(3): 392-4.

3. Cifuentes-Cifuentes Y, Arteaga-Díaz C, Infante-Contreras C, Clavijo-López EG, Quintero-Guzmán C. Prevalencia y caracterización de los recién nacidos con anomalías craneofaciales en el Instituto Materno Infantil de Bogotá. Rev Salud Púb. 2008; 10(3):423-32.

4. Charry I, Aguirre ML, Jaime CCJ, Gómez BJ, Higuera J, Mateus GL, et al. Caracterización de los pacientes con labio y paladar hendido y de la atención brindada en el Hospital Infantil Universitario de Manizalez (Colombia), 2010. Arch Med. 2012; 12(2):190-7.

5. Lei R-L, Chen H-S, Huang B-Y, Chen $\mathrm{Y}-\mathrm{C}$, Chen PK-T, Lee H-Y, et al. Population-based study of birth prevalence and factors associated with cleft lip and/or palate in Taiwan 2002-2009. PLoS One. 2013 Jan; 8(3):e58690.

6. Bedón Rodríguez M, Villota González LG. Labio y paladar hendido: tendencias actuales en el manejo exitoso. Arch Med. 2012; 12(1):107-19.

7. Macintosh RB, Herman LT, Shivapuja PK, Echeverri-Arguello RC. Volunteer cleft surgery in Colombia: An 18-year perspective. J Oral Maxillofac Surg. American Association of Oral and Maxillofacial Surgeons; 2013; 71(10):1742-51.
8. Altamar Ríos J. [Herbicides and congenital malformations in Meta, Colombia]. An Otorrinolaringol Ibero Am. 2002; 29(1):1-20.

9. Altamar-Ríos J. [Meningoencephalocele. A report of 6 cases]. An Otorrinolaringol Ibero Am. 1998 Jan; 25(1):29-38.

10. Fitzsimons KJ, Copley LP, Deacon S $\mathrm{A}$, van der Meulen $\mathrm{JH}$. Hospital care of children with a cleft in England. Arch Dis Child. 2013 Dec; 98(12):970-4.

11. Kobayashi GS, Alvizi L, Sunaga DY, Francis-West $P$, Kuta A, Almada BVP, et al. Susceptibility to DNA damage as a molecular mechanism for non-syndromic cleft lip and palate. PLoS One. 2013 Jan; 8(6):e65677.

12. Acuña-González G, Medina-Solís $C E$, Maupomé G, Escoffie-Ramírez M, Hernández-Romano J, Márquez-Corona MDL, et al. Family history and socioeconomic risk factors for non-syndromic cleft lip and palate: a matched case-control study in a less developed country. Biomédica. 2011; 31(3):381-91.

13. Otero L, Gutiérrez $S$, Cháves $M$, Vargas $C$, Bérmudez L. Association of MSX1 with nonsyndromic cleft lip and palate in a Colombian population. Cleft Palate-Craniofacial J. 2007; 44(6):653-6.

14. Huber JC, Brender JD, Zheng $Q$, Sharkey JR, Vuong AM, Shinde MU, et al. Maternal dietary intake of nitrates, nitrites and nitrosamines and selected birth defects in offspring: a case-control study. Nutr J. Nutrition Journal; 2013 Jan; 12(1):34.

15. Gili JA, Poletta FA, Campaña H, Comas $B$, Pawluk M, Rittler M, et al. Is gravidity $4+$ a risk factor for oral clefts? A case-control study in eight South american 
countries using structural equation modeling. Cleft Palate-Craniofacial J. 2013 Sep; 50(5):591-6.

16. Vila-Nova C, Wehby GL, Queirós F, Chakraborty H, Félix TM, Goco N, et al. Periconceptional use of folic acid and risk of miscarriage: Findings of Oral Cleft Prevention Program in Brazil. J Perinat Med. 2013; 41(4):461-6.

17. Wehby GL, Goco N, Moretti-Ferreira D, Felix T, Richieri-Costa A, Padovani $C$, et al. Oral cleft prevention program (OCPP). BMC Pediatr. BMC Pediatrics; 2012 Jan; 12(1):184

18. Arteaga-Vázquez J, Luna-Muñoz L, Mutchinick OM. Malformaciones congénitas en hijos de madres epilépticas con y sin tratamiento con anticonvulsivantes. Salud Publica Mex. 2012; 54(6):579-86.

19. Castilla EE, Lopez-Camelo JS, Campaña $\mathrm{H}$. Altitude as a risk factor for congenital anomalies. Am J Med Genet. 1999; 86(1):9-14.

20. Mastroiacovo $P$, Maraschini $A$, Leoncini E, Mossey P, Bower C, Castilla E, et al. Prevalence at birth of cleft lip with or without cleft palate: data from the International Perinatal Database of Typical Oral Clefts (IPDTOC). Cleft Palate-Craniofacial J. 2011 Jan; 48(1):66-81.

21. Saxen I. Epidemiology of cleft lip and palate: an attempt to rule out chance correlations. Brit J Prev Soc Med. 1975; 29:103-10.

22. Ochoa Lozano BR, Ortiz de Anda JD, Padilla de la Paz KE, Chacón Martínez $\mathrm{H}$, Blanco Dávila F. Casuística de 10 años de labio y paladar hendido en el Hospital Universitario de la UANL. Med Univ. 2003; 5(18):19-24.

23. García H, Salguero GA, Moreno J, 1810 Arteaga C, Giraldo A. Frecuencia de anomalías congénitas en el Instituto Mater- no Infantil de Bogotá. Biomédica. 2003; 23:161-72.

24. Pachajoa H, Ariza Y, Isaza C. Defectos congénitos mayores en un hospital de tercer nivel en Cali, Colombia 2004-2008. Rev Salud Púb. 2011; 13(1):152-62.

25.Chavarriaga-Rosero J, González-Caicedo MX, Rocha-Buelvas A, PosadaLópez A, Agudelo-Suárez AA. Factores relacionados con la prevalencia de labio y paladar hendido en la población atendida en el Hospital Infantil "Los Angeles", municipio de Pasto (Colombia), 2003-2008. Rev CES Odont. 2011; 24(2):33-41.

26. Cerón Zapata AM, López Palacio AM, Aristizábal Puerta GM, Uribe Álvarez C. A retrospective characterization study on patients with oral clefts in Medellín, Colombia, South America. Rev Fac Odontol Univ Antioq. 2010. p. 81-7.

27. Muñoz J, Bustos I, Quintero C, Instituto E, Naturales DC, Nacional U. Factores de riesgo para algunas anomalías congénitas en población Colombiana. Rev Salud Púb. 2001; 3(3):268-82.

28. Castro Torres AT. Situación de salud para la zona de los llanos y la Orinoquia colombiana [Internet]. Universidad de los Llanos. Villavicencio, Colombia; 2011 [cited 2016 Jan 1]. Available from: http:// web.unillanos.edu.co/docus/SITUACIÓN DE SALUD ORINOQUIA COLOMBIANA 29 DE NOVIEMBRE DE 2011.pdf

29.Cáceres $F$ de $M$, Uscátegui $A M$, Rojas JD, Becerra CH, Díaz LA. Incidencia de las malformaciones congénitas: registro durante un año de vigilancia activa no selectiva en el Hospital Universitario González Valencia. MEDUNAB. 1999; 2(6):109-14.

30. Duque AM, Estupiñán BA, Huertas PE. Labio y paladar fisurados en niños menores de 14 años. Colomb Med. 2002; 33(3):108-12. 
31. Duque AM, Estupiñán $B A$, Huertas PE. Reporte de casos de niños(as) con paladar fisurado aislado, atendidos en el Hospital Universitario del Valle entre 1996 y 2001. Rev Estomatol. 2002; 10(2):57-61.

32. Quijano MC, Rivas J, Salas I, Salazar M, Sánchez B, Sierra CH. Aspectos sociodemográficos y clínicos del labio leporino y paladar fisurado en una población del suroccidente colombiano. Rev Unicauca. 2009; 3:1-10.

33. Zarante I, López MA, Caro A, García-Reyes JC, Ospina JC. Impact and risk factors of craniofacial malformations in a Colombian population. Int J Pediatr Otorhinolaryngol. 2009; 73(10):1434-7.

34. Zarante I, Franco L, López C, Fernández $\mathrm{N}$. Frecuencia de malformaciones congénitas: evaluación y pronóstico de 52.744 nacimientos en tres ciudades colombianas. Biomédica. 2010; 30:65-71.

35. Bermúdez LE. Humanitarian missions in the third world. Plast Reconstr Surg. 2004; 113(1):1687-9. 
INVESTIGACIONES ANDINA No. 34 Vol. 19

\section{Anexos}

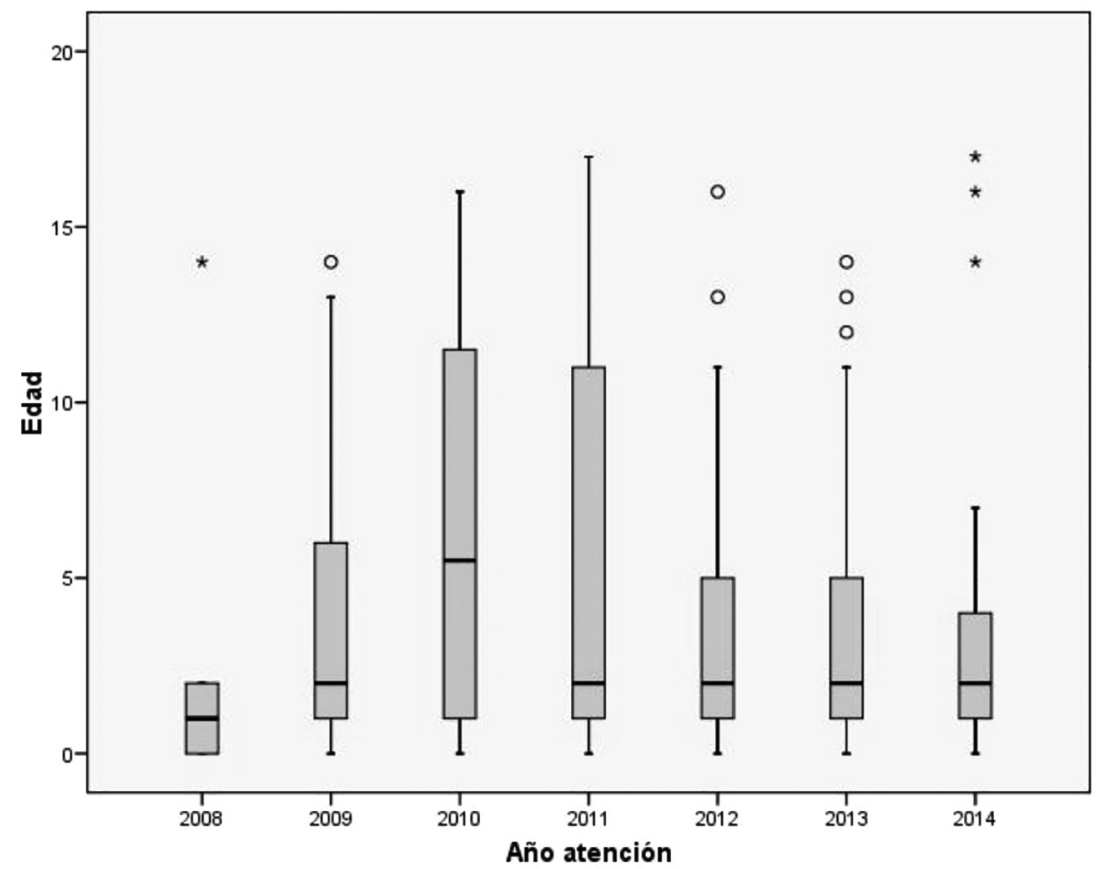

Figura 10. Edad por año del procedimiento en cirugía de labio y paladar hendido en Villavicencio (2008-2014). Los niños operados en el 2010 fueron intervenidos más tardíamente.

Fuente: Elaboración propia. 